\title{
Laboreal
}

Volume $8 \mathrm{~N}^{\circ} 1$ | 2012

Género, Atividades e Saúde

\section{Una investigación pionera en psicopedagogía experimental : introducción al texto de Antoine Léon}

Uma investigação pioneira em psicopedagogia experimental : introdução ao texto de Antoine Léon

Une recherche pionnière en psychopédagogie expérimentale : introduction au texte d'Antoine Léon

An innovative research on experimental psychopedagogy : introduction to

Antoine Léon's text

\section{Serge Blanchard}

\section{(2) OpenEdition}

\section{Journals}

Edición electrónica

URL: http://journals.openedition.org/laboreal/7418

DOI: $10.4000 /$ laboreal. 7418

ISSN: 1646-5237

Editor

Universidade do Porto

\section{Referencia electrónica}

Serge Blanchard, «Una investigación pionera en psicopedagogía experimental : introducción al texto de Antoine Léon », Laboreal [En línea], Volume 8 N¹ | 2012, Publicado el 01 julio 2012, consultado el 23 septiembre 2020. URL : http://journals.openedition.org/laboreal/7418 ; DOI : https://doi.org/ 10.4000/laboreal.7418

Este documento fue generado automáticamente el 23 septiembre 2020

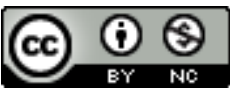

Laboreal está licenciado com uma Licença Creative Commons - Atribuição-NãoComercial 4.0 Internacional. 


\title{
Una investigación pionera en psicopedagogía experimental : introducción al texto de Antoine Léon
}

\author{
Uma investigação pioneira em psicopedagogia experimental : introdução ao \\ texto de Antoine Léon \\ Une recherche pionnière en psychopédagogie expérimentale : introduction au \\ texte d'Antoine Léon
}

An innovative research on experimental psychopedagogy : introduction to

Antoine Léon's text

Serge Blanchard

1 En 1957, Antoine Léon, investigador en el Service de Recherche de l'Institut National d'Etude du Travail et d'Orientation Professionnelle (INETOP), presentó en el Boletín del Centre de Recherche Psychotechnique (CERP), dirigido en ese entonces por Jean-Marie Faverge, una estudio de campo relacionado con "los mecanismos de adquisición, en el adolescente, de los primeros elementos para desarrollar un oficio" [1]. En este artículo (1957a), relataba la investigación realizada en el Centre d'application de l'Ecole Normale Nationale d'Apprentissage (ENNA) de Paris, donde se formaban los profesores técnicos de los Centres d'Apprentissages (CA), establecimientos donde se preparaban durante tres años, con un currículum escolar, los (sus) alumnos para alcanzar la obtención de un Certificado de Aptitud Profesional (CAP). Este texto estaba en correspondencia con su realidad contextual de su época. En los años cincuenta, Francia se encontraba en pleno período de reconstrucción. La demanda de obreros y empleados calificados era grande, y la formación y orientación profesional se volvió una necesidad nacional. En este marco, dos puntos de vista sobre la orientación profesional se oponían : por un lado, el abordaje pronóstico-diagnóstico apoyado históricamente por 
Pieron (1949), director del INETOP, y, por otro lado, la concepción educativa y continua, representada por Antoine Léon (1957b).

2 El que estas perspectivas fueran opuestas pudiera sorprender a los lectores no franceses especialistas en temas laborales. En Francia, la orientación estuvo históricamente vinculada al conocimiento del trabajo. A inicios del Siglo XX, los primeros trabajos sobre orientación tuvieron como objetivo el de regular la entrada de los más jóvenes a un oficio y el de crear formas de acceso a la formación de los trabajadores. Esta cercanía entre ambos objetivos, reivindicada aunque no siempre asumida, permitió comprender que hasta en la actualidad el INETOP reagrupe, en su centro de investigación, equipos de psicología de la orientación, de psicología del trabajo, de ergonomía, de psicodinámica laboral y de formación de adultos [2]. En los albores del Siglo XX, la degradación de las formas de acceder a un oficio y de las condiciones de trabajo de los obreros, hizo de la formación profesional de los jóvenes un elemento central. En este contexto, la idea de orientación profesional que comenzó a desarrollarse estuvo vinculada a la problemática de darles a los jóvenes las ubicaciones laborales en correspondencia con sus aptitudes, de acuerdo a las características propias de cada oficio estudiado. La idea de incluir las características psicológicas en la organización de la distribución de los individuos en el mercado de trabajo no era novedosa, sin embargo, la orientación le confirió, en los primeros años del Siglo XX, una nueva legitimización científica al considerarla como una forma de aplicación de la psicología y de las herramientas que utiliza: los test. Debido a esta voluntad de clasificación, el concepto de aptitud como predisposición natural y las formas de medirlo, fueron considerados esenciales (Ouvrier-Bonnaz, 2007).

\section{La orientación profesional en la Francia de los años $\mathbf{5 0}$}

En Francia, durante los años cincuenta, los consejeros en orientación tuvieron como tarea principal orientar profesionalmente a los alumnos que salían de la escuela primaria a los 14 años y que deseaban continuar con el aprendizaje. El trabajo de los consejeros consistía, esencialmente, en verificar que los jóvenes tuviesen el perfil de aptitudes necesarias en función del oficio escogido. El examen de orientación profesional tenía como objetivo proveer a los jóvenes y a las familias que consultaban a un consejero, las informaciones para ayudarlo en la selección de una profesión o, de forma más frecuente, de una formación profesional. Este examen incluía pruebas escritas que se realizaban en las aulas (englobando los test de razonamiento, de abstracción, de comprensión técnica, de atención, memoria, etc.), al mismo tiempo que se realizaba una consulta individual en el Centre d'Orientation Professionnelle (con una duración de cerca de 2 a 3 horas), que incluía la realización de pruebas de habilidad manual, comprensión técnica, velocidad de reacción...; además de una entrevista donde el consejero debía determinar los intereses, gustos, aspiraciones profesionales del joven, así como el grado de intensidad de las mismas ; y una consulta médica de orientación, realizada por un médico de orientación profesional. El trabajo del consejero se inscribía, por tanto, dentro de los procesos de gestión del diagnóstico de las aptitudes del joven, teniendo por objetivo establecer un pronóstico del éxito en el aprendizaje de una profesión.

4 Reparemos que, al mismo tiempo que se realizaban las tareas de orientación, los consejeros de orientación participaban también en las tareas de selección y de 
reclutamiento de los alumnos en los establecimientos públicos de educación técnica (centros de aprendizaje). Fueron elaborados en el INETOP baterías de test con este propósito, desde 1948. Durante los años 1952-1953 las consultas de alumnos que cursaban los último años de la escuela primaria representaron el $62 \%$ de la actividad de los consejeros de orientación y las consultas concernientes a la entrada a los establecimientos de educación técnica y escuelas profesionales fue del 18 \% (Caroff, 1987, p. 148).

\section{El concepto educativo de Léon en acción dentro de su psicopedagogía de orientación profesional}

5 Es por oposición al proceso de gestión del diagnóstico-pronóstico que Léon desarrolló un concepto educativo sobre la orientación profesional. Léon (1952c) criticó la concepción "reveladora" de la orientación profesional (como fue la utilización del método de los tests) para defender una concepción "formadora". Para Léon era la "noción de la participación activa del niño en la construcción de su futuro profesional lo que caracteriza el concepto educativo en materia de orientación y de adaptación profesional. Este concepto implica una acción educativa continua por parte del maestro, del consejero y de los padres en la preparación del niño para una vida profesional. Esto se sustenta en el carácter histórico, evolutivo de las conductas individuales; en su plasticidad y en el papel decisivo desempeñado, por los medios formadores, en su elaboración. La adaptación profesional, lejos de concebirse en términos de un ajuste mecánico, es consecuencia de las formas de conducta, siempre más eficaces, que el individuo pone en acción para resolver los problemas técnicos y sociales que se le plantean a lo largo de toda su vida" (Léon, 1957b, pp. 12-13). También era necesario "hacer participar activamente a los adolescentes en la elaboración de los proyectos, informarlos para que puedan agrandar su horizonte profesional y puedan escoger su oficio de forma mejor pensada, mejor motivada" (Léon, 1957b, p. 55).

6 León era consciente de los limites de la acción educativa. Sabía que la orientación profesional estaba fuertemente influenciada por el origen social del individuo (ver los resultados de la encuesta del INED acerca de La orientación y selección de los niños en edad escolar, realizada en 1953, por Girard), y por las actitudes del joven y su familia en relación a la escuela. Langevin (1947) reconoció, en este sentido, la impaciencia por dejar la escuela frecuentemente vivenciada por el joven. Nunca subestimó al papel de la cultura general (concepto estrictamente utilitarista de la educación en el medio parental) y al funcionamiento del sistema escolar (organizado, en ese entonces, en dos grandes vías estancas : la primaria y la secundaria), que facilitaba la orientación, de modo precoz, hacia una formación profesional. Por este motivo, su concepto educativo se encontraba inscrito como un proyecto de cambio social (Léon referenció al plan Langevin-Wallon, derivado de la Resistencia, entregado en 1947 en el Gabinete de la Asamblea Nacional y nunca discutido) dirigido a hacer al sistema educativo más democrático y, así, a la sociedad más justa. 


\section{La concepción de Léon con relación al aprendizaje de un oficio por el adolescente}

7 Si bien que el estudio de Léon estuvo focalizado en el tema de los "mecanismos de adquisición, en el adolescente, de los primeros elementos necesarios para un oficio", también se interesó por el conjunto de problemas derivados del tema del aprendizaje de un oficio por el adolescente, elemento direccionado a "responder una serie de problemas que se encuentran a lo largo del periodo que va desde la selección del oficio a la ubicación en la producción" (Léon, 1954, p. 139) :

- Preparación de la elección profesional: junto a sus colegas del INETOP (Bacquet, Cambon, Chaudagne \& Léon, 1957), Léon concibió y puso en práctica diversas sesiones para informar sobre las profesiones, recomendando evaluar los efectos de estas sesiones, específicamente "verificar cómo la información modifica, en el niño, la representación de la vida profesional, cómo se refleja en los diferentes comportamientos, en sus opciones profesionales, en su actitud frente al trabajo escolar" » (Léon, 1957b, p. 66).

- Selección de entrar a los establecimientos de educación profesional : Léon (1957b) criticó la noción de aptitud que daba preponderancia a los factores innatos y que dejaba, por tanto, poco espacio para la posibilidad de realizar intervenciones educativas ; también criticó a los test que proveían una evaluación global del funcionamiento intelectual, pero con poca información acerca de los mecanismos psicológicos desplegados por el joven. Igualmente, criticó el concepto estático de la adaptación del joven-empleo, considerando que tanto el joven como los empleos evolucionaran. Finalmente, destacó la escasa participación del joven en la elaboración de sus proyectos profesionales enmarcados en la gestión de las fases pronóstico-diagnóstico.

- Elecciones profesionales (Léon, 1952a, 1953b) : Léon se interesó por los factores que influencian en la elección de una profesión por parte de los adolescentes como el caso del contenido de la información que se les daba a los mismos (información a los aprendices sobre las perspectivas de futuro de los oficios) y las actividades de pre-aprendizaje de algunos oficios que permitían tener en cuenta el desempeño alcanzado por los adolescentes en el transcurso de los diferentes estadios de iniciación en la profesión. Según Léon, "el negar o, al menos, el descuidar la importancia de la función educativa en la formación de los gustos y las aptitudes profesionales, conduce al psicólogo a orientar su actividad en función de detectar aquello que se supone que sea permanente en el individuo, más que en modificar aquello que resulta un producto transitorio de la serie de interacciones entre el propio individuo y su entorno. La actitud teórica subyacente al control de las previsiones basadas en el empirismo estadístico tiende a limitar las relaciones de orientación profesional y las relaciones escolares a simples intercambios de resultados psicotécnicos o escolares". Para Léon "el consejero debería esencialmente (...) participar de manera directa en el proceso de formación del niño o del adolescente en el marco educativo primario y de educación profesional" (Léon, 1953b, p. 213).

- Inadaptación al aprendizaje (Léon, 1953a): Léon puso el acento en las condiciones de inadaptación inherentes a la vida en las instituciones escolares. Para él, "el conocimiento de estas condiciones permite plantear el problema de la inadaptación más en términos de formación que en términos de selección, más en términos de recuperación que en términos de deficiencias consideradas como inamovibles. Incorpora, al mismo tiempo, las posibilidades de acción del orientador y del educador" (Léon, 1954, p. 150). Léon se interesó también por las condiciones pedagógicas y sociales susceptibles de reforzar la motivación 
para el aprendizaje de una profesión. Si el interés por el oficio aprendido aumentaba entre el primer y el tercer año del CAP, "no se relaciona solamente a una especie de resignación pasiva o a una toma de conciencia del adolescente de la imposibilidad de cambiar de oficio. Está relacionado, especialmente, a la creación de nuevos intereses durante el propio transcurso del aprendizaje del oficio... La evolución de las motivaciones profesionales del adolescente está condicionada por la elaboración de una representación, cada vez más rica y más objetiva, de las condiciones reales del oficio aprendido" (Léon, 1957b, pp. 43-44).

- Docimología de los exámenes profesionales: Léon (1952b) señaló que "la incorporación de exámenes concebidos como medio de control de los conocimientos adquiridos con vistas a determinada formación aparece hoy como legítima y hasta indispensable (...) Las reglas docimológicas solo pueden conservar todo su sentido dentro del marco de reformas importantes, refieriendose respectivamente por la preparación de los candidatos, la elaboración de pruebas y el rol de los examinadores". una psicopedagogía de la orientación con propósito educativo (Léon, 1957b).

\section{Los mecanismos de adquisición en el adolescente de los primeros elementos del oficio}

9 En su "análisis de algunos mecanismos de adquisición en el adolescente de los primeros elementos de un oficio", Léon, en colaboración con Dacquin, profesor de educación técnica, intentó poner en acción su concepto de orientación desde un estudio de campo llevado a cabo con 25 aprendices entre 15-16 años que ya habían frecuentado el primer año de formación en montaje de máquinas y que, al inicio de su segundo año, estaban frecuentando, como becarios, una pasantía de iniciación en el uso de la fresa, por un período de una semana, incluyendo 18 horas de taller.

os aprendices trabajaban en grupos de cuatro, lo que permitió observar sus comportamientos de aprendizaje individuales $\mathrm{y}$, consecuentemente, identificar sus dificultades y sus errores. En función de comprender estos errores, los aprendices son entrevistados tanto de forma directa frente a la maquina, como en el transcurso de un como ejercicio de control. A partir de los análisis de Pacaud (1955), Léon planteó la hipótesis de que determinados errores estaban relacionados a un proceso de generalización apurada y, para verificarla, los aprendices fueron invitados a explicar y justificar por sí mismos tal o cual acción profesional particularmente difícil.

11 Los grupos fueron sometidos a dos métodos educativos diferentes: un método llamado como global-intuitivo y otro en el que los aprendices era invitados a hacer un esfuerzo para analizar y utilizar sus conocimientos generales en la fase preparatoria del trabajo propiamente dicho, sobre la máquina. Apoyándose en los resultados del estudio, Léon defendió el interés y la legitimidad de la investigación psicopedagógica en las áreas de la educación técnica y general, estudio que continuó a lo largo de toda su carrera (Léon, 1977).

\section{¿Qué continuidad tuvo este tipo de investigación ?}

En desacuerdo con su Director H. Pieron (1952), Léon (1991, p. 94) abandonó el INETOP, donde continuaron a desarrollarse investigaciones psicopedagógicas que fueron 
publicadas mucho más tarde, por ejemplo, sobre los temas de la naturaleza y el desarrollo del pensamiento matemático desde el $3^{\circ}$ al $9^{\circ}$ año de escolaridad, por Pelnard, en 1976; sobre la comprensión de la lectura entre el $6^{0}$ y el $9^{\circ}$ año de escolaridad, por Pelnard, en 1981 y Aubret, en 1991; sobre el manejo de máquinasherramientas, por Lemercier, en 1981 ; sobre el pensamiento técnico, por Gillet, de 1979 a 1986 ; sobre la solución de problemas de física, por Rozencwag y Trosseille, en 1996 ; sobre la educabilidad cognitiva, por Loarer, en 1991. En relación a esta última área de investigación, se trató de encontrar soluciones para los problemas de adaptación con que los profesionales poco calificados se encontraban a lo largo de su carrera, debido a las transformaciones tecnológicas y como forma de auxilio a la inserción de poblaciones con baja escolaridad.

Contrariamente a lo propuesto por Léon, los consejeros de orientación se dedicaron poco en el campo de la psicopedagogía de la educación general - excepto en el campo de la detección y el análisis de dificultades de lectura en la entrada al $6^{\circ}$ año de escolaridad (Aubret, Blanchard \& Sontag, 2006) - y técnica, tal vez porque la influencia de las investigaciones psicopedagógicas sobre la formación de los docentes y sus prácticas siempre fueron débiles, incluso en la actualidad. También resulta probable que así fuese porque los trabajos de Léon tuvieron poco eco en los medios de orientación. En Francia, en un momento en que el abordaje educativo de la orientación se instala de forma permanente en las escuelas, la contribución de Léon debería ser todavía útil. De forma general, más allá del contexto francés, en un mundo en el que el trabajo real es cada vez más difícil de ver y de describir y, en consecuencia, de tener en cuenta para ayudar a los jóvenes a una mejor inserción en el mundo profesional ; los trabajos precursores de Léon deberían resultar útiles a todos aquellos que se interesan por la relación a (re)construirse entre el trabajo y su análisis, y la orientación escolar y profesional.

\section{BIBLIOGRAFÍA}

Aubret, J., Blanchard, S., \& Sontag, J.-C. (2006). Évaluer les compétences en lecture des collégiens en $6^{\mathrm{e}} / 5^{\mathrm{e}}$. L'orientation scolaire et professionnelle,35,3, 446-473.

Bacquet, R., Cambon, J., Chaudagne, H., \& Léon, A. (1957). Pour l'information professionnelle des jeunes gens de 14 ans. Paris : Ed. Bourrelier.

Caroff, A. (1987). L'organisation de l'orientation des jeunes en France : évolution des origines à nos jours. Issy-les-Moulineaux, EAP.

Langevin, P. (1947). Numéro spécial : Paul Langevin, écrits philosophiques et pédagogiques. Pour l'Ere Nouvelle, mars-avril.

Léon, A. (1952a). Le choix professionnel des candidats aux centres d'apprentissage. BINOP, numéro spécial.

Léon, A. (1952b). Le rôle des examens. Bulletin de psychologie, numéro spécial, 31-40. 
Léon, A. (1952c). Problèmes théoriques et pratiques de l'orientation professionnelle. La Raison, 4, 122-148.

Léon, A. (1953a). Contribution à l'étude de l'adaptation à l'apprentissage. Enfance, 1, 75-83.

Léon, A. (1953b). Variations des choix professionnels. BINOP, 5, 204-217.

Léon, A. (1954). Quelques aspects de l'apprentissage du métier chez l'adolescent. L'Année Psychologique, 54, 1, 139-156.

Léon, A. (1957a). Analyse de quelques mécanismes d'acquisition des premiers éléments d'un métier chez l'adolescent. Bulletin du CERP, 2, 183-188.

Léon, A. (1957b). Psychopédagogie de l'orientation professionnelle. Paris : PUF.

Léon, A. (1977) (Dir.). Manuel de psychopédagogie expérimentale. Paris : PUF.

Léon, A. (1991). Note sur l'histoire de l'orientation professionnelle en France. Histoire de L'Education, 49, 89-98.

Ouvrier-Bonnaz, R. (2007). A psicologia em França de 1870 a 1940, de uma ciência aplicada a uma disciplina universitária, Laboréal, 3 (1), 57-63. http://laboreal.up.pt/revista/artigo.php

Pacaud, S. (1955). Méthode normative et méthode expérimentale en psychologie du travail. Journal de Psychologie Normale et Pathologique, 52.

Piéron, H. (1949). La psychologie différentielle. Paris : PUF.

Piéron, H. (1952). Le rôle d'un conseiller ne doit pas se confondre avec celui d'un éducateur. BINOP, numéro spécial.

\section{NOTAS}

1. Jean-Marie Faverge, que publicó junto a Ombredane «L'analyse du travail» (1955), encontró innúmeras semejanzas entre los trabajos realizados en el CERP y los de Léon (análisis que se realizaron junto a Jacques Leplat, sucesor de Faverge en la dirección del CERP).

2. El Groupe de Recherche et d'Etude sur L'Histoire du Travail et de l'orientation (GRESHTO), grupo transversal al conjunto de estos equipos, tuvo como objetivo el de pensar en la unión de la historia del trabajo y de la orientación.

\section{AUTOR}

\section{SERGE BLANCHARD}

Groupe de Recherche et d'Etude sur l'Histoire du Travail et de l'Orientation (GRESHTO), Centre de Recherche sur le Travail et le Développement (CRTD), Conservatoire National des Arts et Métiers (CNAM), 41, Rue Gay Lussac 75005, Paris, France

blanchard.serge@wanadoo.fr 